\title{
POTENTIAL PRODUCTION OF ULTRASHORT ELECTRON BUNCHES WITH THE ADVANCED PHOTON SOURCE LINAC*
}

\author{
M. Borland, Advanced Photon Source, Argonne National Laboratory, Argonne, IL ${ }^{\dagger}$
}

\begin{abstract}
With the recent addition of a chicane bunch compressor to the Advanced Photon Source (APS) linac, it may now be possible to create ultrashort electron bunches, with rms bunch lengths on the order of $10 \mathrm{fs}$ and peak currents of several hundred amperes. Although such bunches are too short to be useful for free-electron-laser-related work, they would still provide a potential source of short-pulse spontaneous radiation, either from an undulator or a bending magnet. The APS linac features a high-brightness thermionic rf gun that delivers beam to the linac via an alpha magnet. This setup provides a first stage of bunch compression at an energy of about $3 \mathrm{MeV}$, as well as permitting elimination of the low-energy tail using a scraper in the alpha magnet. In simulations, this beam has been further compressed using the chicane bunch compressor at $150 \mathrm{MeV}$. Scrapers in the chicane are used to aid in the production of a clean, short pulse. This paper discusses the results of these simulations, including jitter sensitivity and the effects of coherent synchrotron radiation and wakefields.
\end{abstract}

\section{INTRODUCTION}

A straightforward way to create ultrashort electron bunches is to combine magnetic bunch compression with beam collimation. Kung et al. [1] reported using the beam from a thermionic rf gun [2][3] and an alpha-magnet [4] [3] for magnetic compression, creating bunches of up to $70 \mathrm{pC}$ with rms bunch lengths of $50 \mathrm{fs}$.

This scheme relies on the nearly monotonic time-energy correlation possible from a thermionic rf gun. This correlation is desirable in a thermionic rf gun because it makes magnetic bunch compression possible. It also allows filtering the beam in time by filtering it in momentum inside the alpha magnet. In this paper, we extend this idea by adding a second stage of magnetic bunch compression following acceleration. This approach has the advantage of allowing shorter pulses to be generated, as well as delaying final compression until the beam is at higher energy, where space charge forces should be greatly reduced.

In this work, we report on simulations of this concept. Of concern is the sensitivity to jitter, particularly in the APS system [5][6], where scraping must be performed in the chicane compressor (because of the lack of a high-energy scraper in the alpha magnet). This situation introduces the

\footnotetext{
* Work supported by U.S. Department of Energy, Office of Basic Energy Sciences, under Contract No. W-31-109-ENG-38.

† borland@aps.anl.gov
}

possibility of large variation in the charge because of jitter in the rf phases, alpha magnet current, and chicane magnet currents.

Fig. 1 shows a schematic of the APS system. The 2,856MHz thermionic rf gun [7] is driven by a dedicated klystron and uses a tungsten dispenser cathode. It is capable of running for long periods with an rf pulse length of $1.5 \mu \mathrm{s}$ and average current of $900 \mathrm{~mA}$, corresponding to a current density of approximately $15 \mathrm{~A} / \mathrm{cm}^{2}$. Because of radiation concerns, an electrostatic deflector (not shown) is used to gate the beam, resulting in a macro-pulse length of $8 \mathrm{~ns}$.

The beam is brought on-axis using an alpha magnet, which can be adjusted to vary the bunch length at the linac entrance. The alpha magnet also has a low-energy scraper, which in the present application is used to remove a significant portion of the beam, leaving only the high-energy core, which has a relatively small emittance.

The beam is accelerated to $150 \mathrm{MeV}$ using four 3-m Sband structures powered by a separate klystron, the phase of which may be adjusted to modify the energy chirp of the beam in order to control subsequent compression in the chicane. The chicane consists of four dipoles and has a pair of scrapers at the center where the dispersion is high. For this application, both the low and high-energy scrapers are needed, even though the scraper in the alpha magnet has removed the low-energy tail. Both are needed because wakefields in the linac may repopulate the low-energy tail, resulting in difficulty making a short bunch. Scraping the low-energy tail in the alpha magnet offers the following advantage: useless particles are dumped at $3 \mathrm{MeV}$ rather than $150 \mathrm{MeV}$. It also speeds the simulation by removing particles early on that will be lost eventually in any case.

Following the chicane are eight more 3-m S-band structures, used for acceleration to as high as $500 \mathrm{MeV}$ and for bunch length measurement using the zero-phasing technique.

\section{SIMULATION METHODS}

The rf gun is simulated using the fully electromagnetic particle-in-cell (PIC) code spiffe [8]. The simulated beam from spiffe contains about 28,000 particles. Because the charge from the gun is only $315 \mathrm{pC}$ per pulse, most of which is scraped away in the alpha magnet, and because the beam energy is $3 \mathrm{MeV}$ at the gun exit, we ignore space charge outside the gun. Tracking from this point on is performed using the 6D tracking code elegant [9], which includes simulation of alpha magnets (with a third-order matrix [3]), rf acceleration with exact phase dependence, 


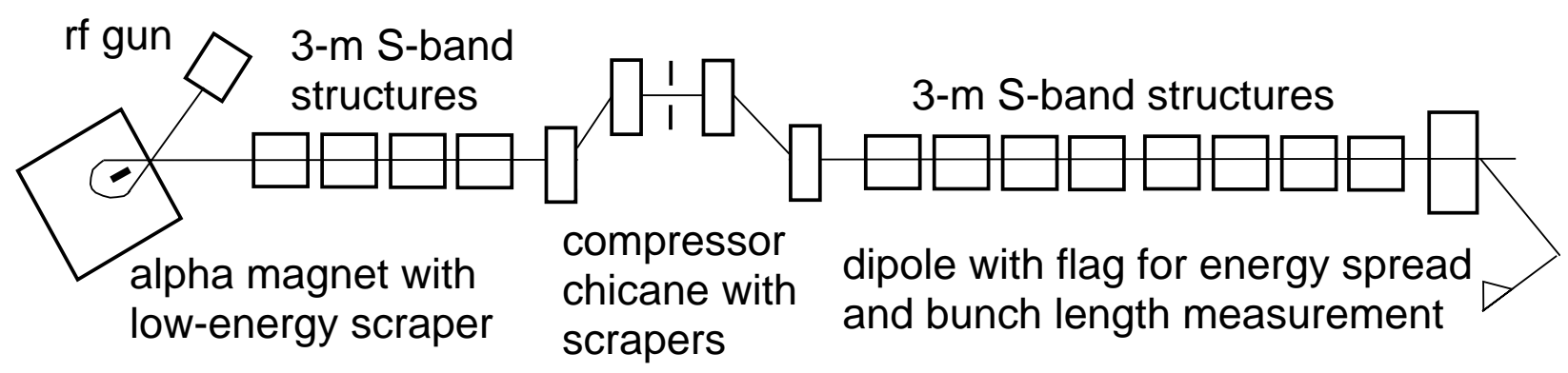

Figure 1: Schematic of APS linac and bunch compressor.

coherent synchrotron radiation (CSR), and wakefields. We used second-order, element-by-element matrix tracking for all other elements (e.g., dipoles, quadrupoles, and drifts).

The simulated beam created by spiffe and tracked through elegant has substantially lower emittance than is measured. The most energetic $50 \%$ of the beam has a measured normalized emittance at the bunch compressor of 10 to $20 \mu \mathrm{m}$, compared to a simulated value of $5.6 \mu \mathrm{m}$. Because the monoenergetic beamsize is very important in determining how well the momentum filtration works, this difference cannot be ignored. Hence, all transverse phasespace coordinates of the beam from spiffe were inflated by the factor $\sqrt{20 / 5.6}$ to match the measured emittance.

The elegant code has the ability to optimize the results of tracking, which is important when the physical effects involved are complex. We wish to produce a very short bunch with as much charge as possible at the end of the linac. We also want to constrain the peak current entering the linac, in order to mitigate emittance growth caused by space charge. The parameters at our disposal are the strength of the alpha magnet, the position of the scrapers in the alpha magnet and chicane, and the phase and voltage of the linac.

Because the momentum filtration is modeled realistically using scrapers that filter the horizontal coordinates at high dispersion points, there is significant sensitivity to the monoenergetic beam size at the scrapers. The final bunch can be lengthened dramatically if the beam is poorly focused at the scrapers. Hence, elegant was also allowed to vary the strength of three quadrupoles upstream of the chicane, in order to allow it to effectively optimize the momentum resolution at the scraper location. To avoid creating unrealistic beam size variations, apertures were introduced upstream and downstream of the chicane, which forced elegant to maintain generally small beam sizes. Of course, the simulations did not proceed in a single step with all of these variables. Rough solutions were obtained with a smaller set of variables, then refined with the full set.

The elegant code was given a target for the average current in the microbunch, defined as $I_{95}=\frac{0.95 * Q}{\Delta t_{95}}$, where $\mathrm{Q}$ is the total charge and $\Delta t_{95}$ is the duration containing the central $95 \%$ of the charge. Target currents between 500 A and 2,000 A were chosen. This approach is preferable to simply minimizing the bunch length, because it provides a well-defined convergence criterion. An upper limit of 30A was placed on the microbunch current entering the linac, and the final rms bunch length was limited to under 25 fs. To avoid making a "beam" consisting of only a few particles, elegant was not allowed to scrape away more than $90 \%$ of the particles. Finally, a beam energy of $150 \mathrm{MeV}$ at the chicane was required. For speed, optimization runs excluded CSR, but included accelerating structure longitudinal wakefields.

Acceleration through the L4 and L5 sections was performed on-crest to $500 \mathrm{MeV}$, because the bunches are so short that there is little hope of removing the energy chirp or canceling wakefields with the rf slope.

\section{SIMULATION RESULTS}

Following optimization, final tracking was executed with CSR in the dipoles and drifts of the chicane. This tracking requires more simulation particles, which were created by replicating each spiffe-generated particle ten times with slight random dithering of the coordinates. We found that CSR had little impact on the beam, probably because the horizontal emittance is already quite large (about 13 $\mu \mathrm{m}$ ), while the charge is low (about $30 \mathrm{pC}$ ). In addition, it is doubtful that the 1-D line-charge formalism used by elegant[10] applies when the bunch is very short compared to its transverse beam size [11], as it is in this case. Hence, CSR was not included in any further simulations.

The optimizer did not obtain $I_{95}$ greater than $930 \mathrm{~A}$, but obtained $\sigma_{t}$ as low as $9 \mathrm{fs}$ and $\Delta t_{95}$ as low as $33 \mathrm{fs}$. Fig. 2 shows the longitudinal phase space for the 930-A result at various points in the system. Note that the momentum cut at the chicane scraper is not very clean, indicating the role of beam emittance and focusing. Also, the peak current in the pulse is about $1,600 \mathrm{~A}$.

\section{JITTER EFFECTS}

This scheme is very sensitive to jitter, particularly in the rf systems. The elegant code was used to simulate this sensitivity for three levels of 2-sigma rms errors. The "base" levels are $1^{\circ}$ phase, $0.2 \%$ voltage, $0.05 \%$ alpha and chicane magnet current, and $7 \%$ gun charge. These levels correspond to present levels for the APS linac. For the "intermediate" level, we divide the phase and voltage jitter 
Table 1: Jitter effects for low- and high-current cases for various error levels.

\begin{tabular}{|l|l||c|c|c|c|c|c|c|}
\hline Case & Error level & $\begin{array}{c}\Delta t_{80} \\
\mathrm{fs}\end{array}$ & $\begin{array}{c}\Delta t_{95} \\
\mathrm{fs}\end{array}$ & $\begin{array}{c}\sigma_{t} \\
\mathrm{fs}\end{array}$ & $\begin{array}{c}\text { Charge } \\
\mathrm{pC}\end{array}$ & $\begin{array}{c}I_{80} \\
\mathrm{~A}\end{array}$ & $\begin{array}{c}I_{95} \\
\mathrm{~A}\end{array}$ & $\begin{array}{c}\sigma_{\delta} \\
\%\end{array}$ \\
\hline \hline low-current & base & $238 \pm 212$ & $304 \pm 257$ & $92 \pm 82$ & $42 \pm 20$ & $212 \pm 136$ & $183 \pm 105$ & $0.12 \pm 0.07$ \\
\hline low-current & intermediate & $76 \pm 43$ & $105 \pm 51$ & $29 \pm 16$ & $34 \pm 10$ & $405 \pm 149$ & $337 \pm 113$ & $0.09 \pm 0.04$ \\
\hline low-current & ultimate & $54 \pm 17$ & $77 \pm 20$ & $21 \pm 6$ & $34 \pm 5$ & $527 \pm 126$ & $435 \pm 92$ & $0.08 \pm 0.02$ \\
\hline high-current & base & $215 \pm 203$ & $282 \pm 249$ & $83 \pm 78$ & $39 \pm 20$ & $258 \pm 230$ & $214 \pm 178$ & $0.11 \pm 0.07$ \\
\hline high-current & intermediate & $63 \pm 45$ & $90 \pm 57$ & $25 \pm 17$ & $32 \pm 12$ & $519 \pm 287$ & $415 \pm 219$ & $0.08 \pm 0.04$ \\
\hline high-current & ultimate & $40 \pm 18$ & $59 \pm 22$ & $16 \pm 7$ & $33 \pm 6$ & $745 \pm 275$ & $586 \pm 201$ & $0.08 \pm 0.02$ \\
\hline
\end{tabular}

by three, while for the "ultimate" level we divide the base phase and voltage jitter by 10 , giving $0.1^{\circ}$ and $0.1 \%$, respectively. The effect of the beam moving relative to the scrapers is included.

For each error level, 500 randomized simulations were done for two configurations from the optimizer, a "low current" case giving (in the absence of jitter) $\Delta t_{95}=62 \mathrm{fs}$, $\sigma_{t}=16 \mathrm{fs}$, and $I_{95}=520 \mathrm{~A}$, and a "high current" case giving $\Delta t_{95}=34 \mathrm{fs}, \sigma_{t}=9 \mathrm{fs}$, and $I_{95}=930 \mathrm{~A}$. Table 1 shows statistics for the jitter runs, while Fig. 3 shows histograms for the high-current case.

Correlation analysis of the base level data shows that precompressor linac voltage jitter causes about $20 \%$ of the variation in rms bunch length. Jitter in precompressor linac and gun phase and in gun voltage each contribute 1-8\%. Hence, there is no dominant jitter source and stabilization requires improvements in most of the systems.

\section{CONCLUSION}

The simulations indicate that, with realistic improvements in rf system stability, it is possible to reliably produce electron bunches with rms bunch lengths of 10-20 fs for bunches containing $33 \mathrm{pC}$. Factors omitted from the simulations that might affect the results are wakes from the scrapers and space charge effects outside the gun.

\section{REFERENCES}

[1] P. H. Kung et al., Phys. Rev. Lett. 73 (1994) 967-970.

[2] M. Borland et al., "Performance of the $2-\mathrm{MeV}$ Microwave Gun for the SSRL $150 \mathrm{MeV}$ LINAC," Proc. of Linac 1990, Albuquerque, NM, 1991.

[3] M. Borland, "A High-Brightness Thermionic Microwave Electron Gun," SLAC-R-402, February 1991.

[4] H. A. Enge, Rev. Sci. Instrum. 34 (1963) 385.

[5] G. Travish et al., "High-Brightness Beams from a Light Source Injector,” Proc. of Linac 2000.

[6] M. Borland et al., "A Highly Flexible Bunch Compressor for the APS LEUTL FEL,” Proc. of Linac 2000.

[7] M. Borland, "An Improved Thermionic Microwave Gun and Emittance-Preserving Transport Line," Proc. of 1993 PAC, Washington, 3015-3017.
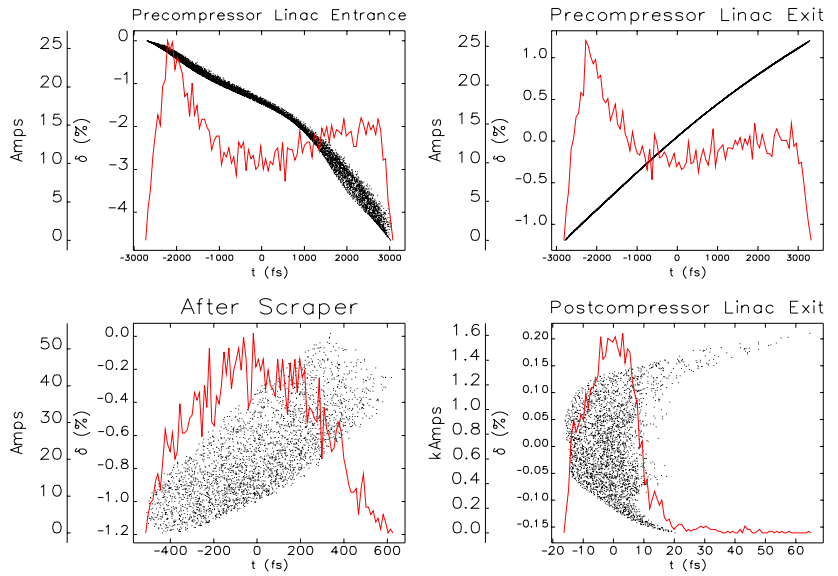

Figure 2: Longitudinal phase space evolution for 930-A average current.
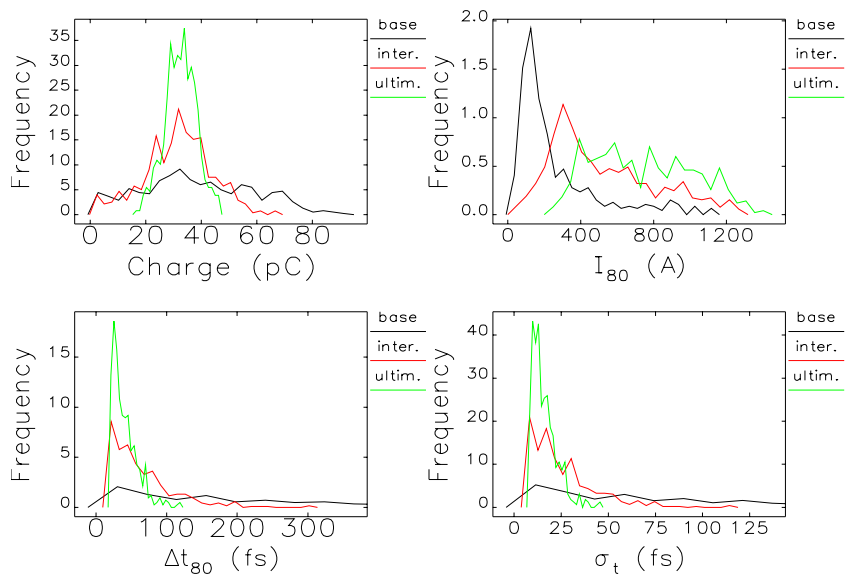

Figure 3: Jitter results for high-current case.

[8] M. Borland, unpublished program.

[9] M. Borland, APS LS-287, September 2000. http://www.aps.anl.gov/techpub/lsnotes/lsnotesTOC.html

[10] M. Borland, "Simple Method for Particle Tracking with Coherent Synchrotron Radiation,” PRST-AB, to be published.

[11] P. Emma, private communication. 